\title{
Microbiome in Health: Establishment, Metabolism, Immunity and Neuronal Pathway
}

\author{
Matthew Obaineh Ojezele ${ }^{1}$ Simon Irikefe Ovuakporaye ${ }^{2}$,Emmanuel Adesola \\ Adedapo $^{3}$
}

${ }^{1}$ Department of Pharmacology and Therapeutics, Delta State University, Abraka, Nigeria.

${ }^{2}$ Department of Human Physiology, Delta State University, Abraka, Nigeria

${ }^{3}$ Department of Microbiology, University of Ibadan, Ibadan, Nigeria

\section{ABSTRACT}

\begin{abstract}
The studies on microbiome encountered a blast, lately, as scientists become mindful of the role of microbiota in the advancement of specific kinds of maladies. The human microbiome is described as a community of microorganisms of different taxa colonizing the human body; this includes the metagenomics and metabolomics of these organisms. Humans have customized microbiome in terms of distribution and composition which are partly determined by host genotype as well as the initial colonization which takes place after delivery. The human gut microbiome has a vital influence on immunity and how it responds to body signals, which is very important for the lymphoid tissue growth, maintenance, and regulation of intestinal immunity. This review aimed at providing an overview of the role of the human microbiome in health spanning the development of the microbiome in utero to postnatal period.
\end{abstract}

Keywords: Blood-brain-barrier; Immunology; Homeostasis; Microbiome

\section{Correspondence:}

Dr Simon Irikefe Ovuakporaye

Department of Human Physiology,

Delta State University,Abraka, Nigeria

ORCID ID: 0000-0001-6350-5864

Email: simonovuakpo2006@yahoo.com

Submitted: $29^{\text {th }}$ August 2020

Accepted: $18^{\text {th }}$ December 2020

Source of Support: None

Conflict of Interest: None

Citation: Ojezele MH, Ovuakporaye SI, Adedapo EA. Microbiome in Health: Establishment, Metabolism, Immunity and Neuronal Pathway. NMJ 2020;3(2):37983. DOI $10.3126 / \mathrm{nmj} . \mathrm{v} 3 \mathrm{i} 2.30925$

\section{INTRODUCTION}

The investigations on the microbiome encountered a blast, lately, as scientists become mindful of the role of microbiota in the advancement of specific kinds of maladies. ${ }^{1}$ The microbiome can be described in at least three dimensions of definition. The first is that used in most biomedical research: the microbial organisms on a defined anatomical space usually limited to bacteria. The second definition incorporates energetics and digestion: the information sources and yields of a particular microbial group. The third definition has the microbial ecology viewpoint that includes the host and microbiome. ${ }^{2}$ There may be the need to define microbiomes during scientific investigations with more exactness as some of the techniques used present indeterminable heterogeneity in the microbiome sampled. For instance, rectal swabs or voided feces are the common samples collected when dealing with the gut microbiome. However, this is largely different from direct sampling of the stomach's microbiome or intestinal and excreta which are likely to affect undefined and potentially variable biosamples; whereas, the ideal analysis should require complex spatial structures of the gut and intestinal microbiome. ${ }^{2}$ 
The human gut is an ecosystem to about $3.8 \times 10^{13}$ microorganisms achieving a total weight of about $1.8 \mathrm{~kg}$, collectively named gut microbiota, which is perceived to regulate the host physiology and health by applying key functions, spreading over from metabolic to immunomodulatory properties. To perform these tasks, the microbiota establishes equilibrium with the host, a condition referred to as eubiosis. ${ }^{3}$ As of now, the components of co-operations or safeguard against potential pathogens are regularly portrayed at the molecular level.

The advent of culture-independent molecular techniques which includes areas such as metagenomics, transcriptomics, proteomics, and metabolomics has eradicated the caveats placed by culture-dependent procedures. ${ }^{4}$ Likewise, it has changed the status quo of the prior knowledge of the human microbiome in health and disease. The changing aspects of the human microbiome are involved in some human gastrointestinal and nongastrointestinal maladies making it a current issue in biological and medical sciences. In addition, certain applications were developed for the human microbiome such as faecal microbiome therapy, antimicrobial discovery, prebiotics, probiotics, and phage therapy to mention a few. A certain number of factors have been identified to affect the host-microbiome such as lifestyle, stage of life, occupation, diet, mode of delivery, and environment. There is a need, however, for more investigations to elucidate the physiological functions of the human microbiome. Consequently, this review aimed at providing an overview of the role of the human microbiome in health spanning the development of the microbiome in utero to postnatal.

\section{OVERVIEW OF THE MICROBIOME}

The human microbiome is described as a community of microorganisms of different taxa colonizing the human body (bacteria, fungi, archaea, viruses, and protozoa); this includes the genome composition (metagenomics) and metabolites (metabolomics) of these organisms. This description also includes microorganisms colonizing the skin, mouth, ears, lungs, and the gut with much emphasis on bacteria especially the gut microbiome; however, there is a shift into studying other groups of organisms such as fungi and viruses. Generally, irrespective of the site of colonization, the classes of human microbiome include the Actinomycetes, Proteobacteria, Firmicutes, Fusobacteria, Cyanobacteria, Verrucomicrobia, and the Bacteroidetes. ${ }^{1,5-7}$

Microbiota varies in distribution based on their location in the gastrointestinal tract. Due to the production of acid in the stomach and production of pancreatic enzymes in the duodenum, microbiome density in these areas is relatively low. ${ }^{7,8}$ In a study, Firmicutes, Bacteroidetes, and Proteobacteria were the most frequently identified bacteria in a healthy lung with minimal involvement of common pathogenic Proteobacteria including Haemophilus. Conversely, there is a modification in the microbial composition in smokers ${ }^{9}$ affecting mainly the Firmicutes and Neisseria species as well as a reduction in the relative abundance of Proteobacteria; a modification which is irreversible after giving up smoking. ${ }^{10}$

On the other hand, in the distal small intestine, there is a progressive increase while the colon microbiome density reaches its highest with anaerobes being predominant. The microbiome has been singled out for its defensive role against invading pathogens as more than $10^{14}$ microorganisms are distributed throughout the entire surface of the digestive tract primarily competing for nutrients and space with pathogenic microbes, producing inhibitory substances and preventing their entry into the intestinal lumen. ${ }^{8}$

\section{MICROBIOME ESTABLISHMENT}

The intrauterine environment has been previously thought to be sterile until molecular techniques detected commensal bacteria in the amniotic fluid and placentas of healthy infants suggesting an exchange of microorganisms between the mother and the foetus. ${ }^{11,12}$ Furthermore, it has been reported that the meconium microbiota of infants delivered through caesarean section has $55 \%$ bacterial homology with the placenta and amniotic fluid microbiota. ${ }^{11,12}$ It has been established that humans have customized microbiome in terms of distribution and composition which is determined in part by host genotype as well as the initial colonization which takes place after delivery. ${ }^{5,8}$

Many factors have been identified to determine the ultimate alterations in the microbial pattern in a particular host; this includes the type of delivery, breastfeeding, lifestyle, diet, hygienic environmental conditions, use of antibiotics, and vaccination. ${ }^{8,13}$ Postnatal bacterial colonization of infants begins almost immediately as the infant has been exposed to the maternal faecal and vaginal microbiota which spreads within 24 hours of delivery. ${ }^{12,13}$ While the various body sites (oral, skin, meconium, e.t.c) of a caesarean delivered infant is initially colonized by bacteria from the mother's skin usually Staphylococcus spp., infants delivered through the vagina are colonized by vaginal bacteria consisting of Prevotella, Attopobium spp., Bacteroides, Escherichia, as well as Shigella. ${ }^{12,13}$ Three days postnatal, infants are colonized by Enterobacteriaceae in abundance while an alteration could be observed at six months of age with an abundance of Bifidobacterium and Collinsella and decreased levels of Streptococcus and Enterobacteriaceae. In the space of three years, infants' gut microbial population closely mimics that of the adults composed of Gram-negative Bacteroidetes and Gram-positive Firmicutes while other species such as Proteobacteria, Actinobacteria, and Fusobacteria are present but few in population. ${ }^{5}$ It has been observed that each individual has a unique microbial composition, a notion which has been proposed that microbial fingerprints might become a tool for personal identification. $^{5}$

Breastfeeding provides key energy sources for many bacteria. Findings showed that infants who were breastfed in the first $30-$ 40 days received an average of $28 \%$ maternal bacterial and $10 \%$ from maternal areolar skin. ${ }^{12}$ More so, infants that are breastfed are observed to have a high population of Bifidobacteria and Lactobacillus spp. while formula-fed infants have a high population of Clostridiales and Proteobacteria. Furthermore, formula-fed infants show decreased bacterial diversity and richness even after one or two years of life. Besides, the ingestion of a high-fat diet during pregnancy and breastfeeding was reported to bring about dysbiosis. ${ }^{12}$ 


\section{MICROBIOME AND IMMUNITY}

The microbiome has an influence on many physiological processes including the development of the immune system, processing polysaccharides from diets, $\mathrm{pH}$ regulation, vitamin and hormone production, processing, and detoxification of environmental chemicals as well as the maintenance of the skin and mucosal barrier function. ${ }^{7}$ The normal human gut mucosa is able to filter commensal microorganisms from pathogenic organisms through the expression of pattern recognition receptors which includes Nucleotide-Binding Oligomerization containing proteins (NOD) present in the cytoplasm, RIG-1 like helicases, and Toll-Like Receptors (TLRs) on the cell surface which activate the nuclear factor $\kappa \mathrm{B}(\mathrm{NF} \kappa \mathrm{B})$ group transcription factors with the possible resultant induction of more than 100 pro-inflammatory and host response genes. ${ }^{13,14}$ The human gut microbiome has a vital influence on immunity and how it responds to body signals, which is very important for the development of lymphoid tissues, maintenance, and regulation of intestinal immunity. ${ }^{8}$

Studies based on germ-free mice show that bacterial colonization is the basis for the gut-associated lymphoid tissues (GALT), as well as the formation of B-cell IgM+4 antibodies. ${ }^{7,8}$ The intestinal mucosa of these germ-free animals had lower B, T, and dendritic cells count; immature mesenteric lymph nodes, and Peyer's patches. ${ }^{8}$ Furthermore, their villi were observed to be longer and narrower; the cavities were also observed to be deeper. However, after the colonization of these mice with commensal microorganisms, their immune systems improved and normalized. ${ }^{7,8}$ The $\mathrm{T}$ cells have been observed to generate subpopulations whose immune responses may be anti or proinflammatory which is essential against food proteins or intestinal microbiota. ${ }^{8}, 15$ Bacteroides fragilis, a bacterium whose capsule contains polysaccharide A has been observed to induce CD4+ $\mathrm{T}$ cells into regulatory $\mathrm{T}$ cells favouring the production of antiinflammatory cells as well as transforming growth factor-beta (TGF- $\beta$ ); neutralizing the pro-inflammatory response of Th17 (T helper) cells. ${ }^{8}$

Conversely, filamentous bacteria have been observed to be capable of inducing pro-inflammatory cells (Th17 T helper cells); mice with no filamentous bacteria have been observed to have a very weak immune response and therefore become more susceptible to infections. Based on these observations, it has been suggested that immunological responses triggered by the intestinal microbiota can strengthen the intestinal barrier. ${ }^{8,15}$ These findings suggest that there is an established mutualistic interaction that exists between the microbiota and immune system of the host resulting in myriads of immunological responses such as the production of IgA and antimicrobial peptides which allow maintenance of a dynamic balance with the commensal microorganisms. ${ }^{8,11}$ Further findings confirmed the initial hypothesis that a decrease in microbial population caused by the improvement of hygienic conditions may have largely contributed to the increased prevalence of autoimmune diseases in the developed countries. ${ }^{8}$

\section{MICROBIOME AND METABOLISM}

There is an existing mutualistic relationship between the host cells and the microbiome which exists as a two-way dialogue that centres on metabolism as a central theme; an association referred to as 'Amphibiosis'..$^{5,7,13}$ The human microbiome plays a direct role in nutrient metabolism and vitamins (group B and $\mathrm{K}$ ) that are intrinsically important for host sustainability, working together to derive energy from food. This energy is obtained during the process of fermentation of non-absorbable carbohydrates which brings about the production of short-chain fatty acids (SCFAs) such as butyrate, propionate, and acetate; hydrogen and carbon dioxide. ${ }^{7,13,16}$

Butyrate is required by human enterocytes, especially the colonocytes as their main source of energy. It is capable of inducing apoptosis of colon cancer cells, activates intestinal gluconeogenesis, and has a beneficial effect on glucose-energy balance. Furthermore, butyrate is essential for the consumption of large amounts of oxygen through $\beta$ oxidation process thereby, creating a state of anoxia that sustains oxygen equilibrium and as a result, prevents microbiota dysbiosis. ${ }^{7,17,18}$

Propionate regulates gluconeogenesis and satiety signalling through the interaction with fatty acid receptors in the liver while acetate is an important metabolite for other bacterial growth; it is used in the metabolism of cholesterol and lipogenesis and plays a central role in appetite regulation. ${ }^{17,18}$ Other products include trimethylamine obtained from dietary phosphatidylcholine and carnitine from meat and dairy which depends on the gut microbiota. It is oxidized to trimethylamine $\mathrm{N}$-oxide in the liver which is associated with the risk of atherosclerosis. Indolepropionic acid is highly associated with fibre intake and has been found to have a very strong radical scavenging activity in vitro which reduces the risk of type 2 diabetes. ${ }^{18}$

Fatty acids (SCFAs) acting as ligands, trigger the activation of G-protein coupled receptors: GPR41 and GPR43, exhibited in several cells such as the immune, endocrine, and adipose cells (Mehajeri et al., 2018). This activation process is essential for the expression of YY peptide and glucagon-like peptide 1 (GLP1) production which contributes to the inhibition of the lipolytic process and adipocyte differentiation resulting in weight gain (increased adipose tissue) in animals. ${ }^{8,17}$

It has been observed that butyrate supplementation to obese, prediabetic mice improved the intestinal epithelial barrier and insulin secretion from $\beta$ cells and decreases body adiposity as weight gain, insulin resistance, hyperinsulinaemia and hyperglycaemia. ${ }^{17}$ The SCFAs prevent the growth of bacterial pathogens through the acidification of the lumen in the colon. ${ }^{7,8}$ Gut microbiota is also involved in the metabolism of bile acids from dietary cholesterol as primary bile acids bind to receptors, promoting fat and fatsoluble vitamins absorption, and bind to cell receptors such as the TGR-5 triggers several metabolic effects such as resistance to weight gain and prevention of hepatic steatosis.

This bile acid metabolic process is controlled by gut microbial enzymes. ${ }^{8,18}$ In addition, certain clades of bacteria such as the Bacteroidetes and Firmicutes have been shown to improve ATPbinding cassette transporter expression in enterocytes and GLP-1 and GLP-2 secretion. ${ }^{17}$ Fatty acids (SCFAs) have been noted to exert multiple effects on the host which include but not limited to acting as an energy source, maintenance of glucose and energy homeostasis, regulation of anorectic hormones that play vital roles in appetite control, tumour suppression (butyrate) as well as regulating central and peripheral nervous systems. ${ }^{19}$ 


\section{MICROBIOME AND GUT-BRAIN RELATIONSHIP}

Two-Way directional signalling exists between the intestinal microbiota, the gut and the brain. This exists along neuronal pathways involving the central and enteric nervous systems in addition to the circulatory system. ${ }^{20}$ The central and enteric nervous systems include the involvement of the hypothalamic-pituitaryadrenal (HPA) axis, immune system regulators, hormones, bacterial metabolites (SCFAs and tryptophan, and bile acids), and neurotransmitters such as serotonin, dopamine, noradrenalin, and 5-hydroxytryptamine produced from tryptophan conversion. The gut microbial generation of neurotransmitters such as serotonin has made researchers presume the gut microbiome as the "second brain', ${ }^{5,21}$

Gut microbiota produces SCFAs which circulate and influence the integrity of the blood-brain barrier (BBB) and triggers the production of the tight junction proteins claudin-5 and occludin. This influence on the BBB occludes the entry of undesired metabolites into the brain. ${ }^{16,20}$ Lipopolysaccharide, bacterial lipoprotein, flagellin and $\mathrm{CpG}$ islands of unmethylated DNA which are collectively called microbe-associated molecular patterns which are produced by the gut microbiota affect neuroimmune function by triggering the secretion of cytokines ( $\mathrm{TNF} \alpha$, IL-6, and IL-1 $\beta$ ) from innate immune cells (dendritic cells, macrophages, and neutrophils). This crosses the BBB, activating the microglia and neurons resulting in transformed neurological functions which can lead to mood and behavioural changes.

Furthermore, the administration of probiotics containing Bifidobacterium animalis, sub-sp. lactis, Streptococcus thermophiles, and two Lactobacillus spp. have been shown to exert significant brain activity in healthy women assessed by functional magnetic resonance imaging (fMRI). ${ }^{22}$ This was in response to an emotional faces attention mask as reduced fMRI reactivity was found in interceptive and somatosensory regions of the brain controlling the central processing unit of emotion and sensation. Likewise, probiotics containing Lactobacillus helveticus and Bifidobacterium longum have also shown beneficial mental effects in healthy humans with substantial improvements in several global tests which include reduction of global psychological symptoms, depression, and anxiety. ${ }^{23}$

\section{CONCLUSIONS}

Even though researchers have and are still unravelling the potentials of the human microbiome and its effect on health, we have been able to understand how the microbiome is an intrinsic part of human existence. Microbiota, in general, is not passive in their interaction with their human hosts. They are essential in the early life of an individual as they spell out the health conditions that are likely to surface as a child grows to adulthood. More so, they participate actively in the maintenance of the immune response to pathogens, thereby becoming a member of the adaptive immunity of the host. Furthermore, their role in diet metabolism cannot be overemphasized as they are involved in energy production, emulsification processes, regulation of glucose level in the body in conjunction with the pancreas, and maintenance of body adiposity to regulate weight gain in humans. Also, their role in the secretion of neurotransmitters is notable as this will be beneficial to correct many ailments that might have led to mental disorders due to under secretion of these neurotransmitters.

\section{REFERENCES}

1. Cani DP. Human gut microbiome: hopes, threats and promises. Open Press BMJ. 2018; 67:1716-25. Crossref

2. Silbergeld KE. The microbiome: modulator of pharmacological and toxicological exposures and responses. Sage Toxicologic Pathology. 2017; 45(1) 190-4. Crossref

3. Panebianco C, Angello A, Valiero P. Pharmacomicrobiomics: exploiting the drug-microbiota interactions in anticancer therapies. BMC Microbiome; 2018; 6:92. Crossref

4. Edet UO, Antai SP, Brooks AA, Asitok AD, Enya O, Japhet FH. An overview of cultural, molecular and metagenomic techniques in description of microbial diversity. Journal of Advances in Microbiology. 2017; 7(2): 1-19. Crossref

5. Swanson HI. Drug metabolism by the host and gut microbiota: a partnership or rivalry? Drug Metabolism and Disposition. 2015; 43: 1499-504. Crossref

6. O'Dwyer ND, Robert PD, Bethany BM. The lung microbiome, immunity and the pathogenesis of chronic lung disease. The Journal of Immunology. 2016; 196:4839-47. Crossref

7. Scotti E, Stéphanie B, Giuseppe LS, Filippo Z, Vincenzo B, Carine $\mathrm{P}$, et al. Exploring the microbiome in health and disease: implications for toxicology. Toxicology Research and Application. 2017; 1: 1-32. $\underline{\text { Crossref }}$
8. Passos MCF, Moraes-Filho JP. Intestinal microbiota in digestive disease. Arq Gastroenterol. 2017; 54 (3): 255-62. $\underline{\text { Crossref }}$

9. Ojezele MO, Ehwarieme DA, Atube M, Potokiri A. Effects of caffeine and nicotine on the population and diversity of gut bacterial microbiome of adult Wistar rats. Nigerian Journal of Science and Environment. 2017; 15 (1):141-6. Website

10. Faner R, Oriol S, Alvar A, Eric B, James DC, Gary BH, et al. The microbiome in respiratory medicine: current challenges and future perspectives. Eur Respr J. 2017; 49:1602086. Crossref

11. Lynch VS, Pederson O. The human intestinal microbiome in health and disease. The New England Journal of Medicine. 2016; 375:236979. Crossref

12. Stiemsma LT, Michels KB. The role of the microbiome in the developmental origins of health and disease. Paediatrics. 2018; 141(4): e20172437. Crossref

13. Torres MI, Palomeque T, Lorite P. New aspect of Crohn's disease pathogenesis: dysbiotic gut microbiome. Group SM; 2016. Website

14. Sullivan A, Eoin H, John M, Desmond MM. The microbiome and the pathophysiology of asthma. Respiratory Research. 2016; 17:163-70. Crossref

15. Kirby OT, Ochoa-Repáraz. The gut microbiome in multiple sclerosis: a potential therapeutic avenue. Medical Sciences. 2018; 6:69-79. Crossref 
16. Mohajeri MH, Robert JM, Brummer RA, Rastall RK, Weersma HJM, Harmsen MF, Manfred E. European Journal of Nutrition. 2018; 57 (Suppl 1): S1-S14. $\underline{\text { Crossref }}$

17. Sohail UM, Asmaa A, Haseeb A, Roberto R, Hany EM. Role of the gastrointestinal tract microbiome in the pathophysiology of Diabetes Mellitus. Journal of Diabetes Research 2017; Article ID 9631435, Crossref

18. Valdes AM, Walter J, Segal E, Spector TD. Role of the gut microbiota in nutrition and health: discuss strategies from modulating the gut microbiota through diet. Science and Politics of Nutrition 2018; $361: \mathrm{k} 2179$ Crossref

19. Wu X, Wu Y, He L, Wu L, Wang X, Liu Z. Effects of the intestinal microbial metabolite butyrate on the development of colorectal cancer. J. Cancer. 2018; 9(14):2510-2517. $\underline{\text { Crossref }}$
20. Carabotti M, Scirocco A, Maselli MA, Severi C. The gut-brain axis: interactions between enteric microbiota, central and enteric nervous systems. Ann Gastroenterol. 2015; 28(2):203-9. Website

21. Ochoa-Repáraz J, Kasper LH. The second brain: is the gut microbiota a link between obesity and Central Nervous System disorders? Curr. Obes. Rep. 2016; 5: 51-64. Crossref

22. Fijan S. Microorganisms with claimed probiotic properties: an overview of recent literature. Int. J. Environ. Res. Public Health. 2014; 11(5):4745-67. Crossref

23. Messaoudi M, Violle N, Bisson JF, Desor D, Javelot H, Rougeot C. Beneficial psychological effects of a probiotic formulation (Lactobacillus helveticus R0052 and Bifidobacterium longum R0175) in healthy human volunteers. Gut Microbes. 2011; 2(4):25661. $\underline{\text { Crossref }}$ 